\title{
Total-Scattering Pair-Distribution-Function of Organic Material from Powder Electron Diffraction Data
}

\author{
Tatiana E. Gorelik ${ }^{\mathrm{a}}$, Martin U. Schmidt ${ }^{\mathrm{b}}$, Ute Kolb ${ }^{\mathrm{a}, \mathrm{c}}$, and Simon J. L. Billinge ${ }^{\mathrm{d}}$ \\ a Institute of Physical Chemistry, Johannes Gutenberg-University, Jakob Welder Weg 11, \\ 55128 Mainz Germany, gorelik@uni-mainz.de, kolb@uni-mainz.de \\ ${ }^{\mathrm{b}}$ Institute of Inorganic and Analytical Chemistry, Goethe-University, Max-von-Laue-Str. \\ 7, D-60438 Frankfurt am Main, Germany, m.schmidt@chemie.uni-frankfurt.de \\ c Institute of Applied Geosciences, Technical University Darmstadt, Schnittspahnstr. 9, \\ 64287 Darmstadt, Germany, kolb@geo.tu-darmstadt.de \\ ${ }^{\mathrm{d}}$ Department of Applied Physics and Applied Mathematics, Columbia University, New \\ York, NY 10027, USA and Condensed Matter Physics and Materials Science Department, \\ Brookhaven National Laboratory, Upton, NY 11973, USA. Sb2896@columbia.edu
}

\section{Abstract}

This paper shows for the first time that pair-distribution function analyses can be carried out on organic and organo-metallic compounds from powder electron diffraction data. Different experimental setups are demonstrated, including selected area electron diffraction (SAED) and nanodiffraction in transmission electron microscopy (TEM) mode or nanodiffraction in scanning transmission electron microscopy (STEM) mode. The methods were demonstrated on organo-metallic complexes (chlorinated and unchlorinated copper-phthalocyanine) and on purely organic compounds (quinacridone). The PDF curves from powder electron diffraction data, called ePDF, are in good agreement with PDF curves determined from X-ray powder data demonstrating that the problems of obtaining kinematical scattering data and avoiding beam-damage of the sample are possible to resolve.

\section{Introduction}

Amorphous and nanocrystalline materials, including nanostructured bulk and nanoporous materials, have different properties than crystalline bulk materials. This holds not only for 
inorganic compounds, but also for organic or organometallic materials and metal complexes. Examples include organic pigments (where the colour depends on the crystallite size) (Herbst \& Hunger, 2004), organic semiconductors (where the optical and electrical properties depend on the crystallinity), and pharmaceutical compounds, where nanocrystalline and amorphous materials generally show increased solubility and bioavailability (Kim et al., 2008, Yu, 2001). Several active pharmaceutical ingredients (APIs) are industrially produced as nanocrystalline or amorphous powders (Prasad et al., 2010) through cryomilling, melt extrusion, spray drying or rapid precipitation in the presence of crystallization inhibitors. Some APIs are produced and distributed in amorphous forms because they cannot be crystallized at all. The properties of all these amorphous (or nanocrystalline) materials depend strongly on their synthesis or processing conditions.

Structural characterization of inorganic amorphous materials may be carried out using the pair-distribution function, PDF (Warren 1969, Egami \& Billinge 2012). The PDF $G(r)$ represents the probability of finding a pair of atoms with an interatomic distance $r$, weighted by the scattering power of the individual atoms (Egami \& Billinge, 2012, Farrow \& Billinge, 2009). Such an approach is equally applicable to amorphous and nanostructured organic materials, as recently demonstrated (Bates et al., 2006, Schmidt et al., 2009, Billinge et al. 2010, Dykne et al. 2011) where for a molecular crystal, the PDF profiles include intramolecular distances as well as the interatomic distances between different molecules.

The PDF, $G(r)$, can be obtained from powder diffraction data after proper normalization and corrections to obtain the structure function S(Q) (Warren 1969, Egami \& Billinge 2012, Farrow \& Billinge 2009) according to

$$
G(r)=\frac{2}{\pi} \cdot \int_{Q_{\min }}^{Q_{\max }} Q \cdot[S(Q)-1] \cdot \sin (Q r) d Q,
$$

where $Q$ is the magnitude of the scattering vector. The kernel of the Fourier transform, $Q[S(Q)-1] \cdot$, is often referred to as the reduced structure function, $F(Q)$, where

$$
Q=\frac{4 \pi \sin \Theta}{\lambda}
$$

The structure function $S(Q)$ is experimentally obtained through a powder diffraction measurement. It is also known as the total scattering structure function because it is collected over a wide range of reciprocal space and includes not only the Bragg reflections (if present), but also the scattering information between them (Egami and Billinge 2003), i.e., it utilizes all the coherent scattering from the sample. 
PDF analysis has been a standard tool for the investigation of inorganic liquids, glasses and disordered inorganic materials for decades (see e.g. Warren 1969, Klug \& Alexander, 1974, Wagner 1978, Waseda, 1980, Wright 1985, Barnes et al., 2003, Neder \& Proffen, 2009). In recent years the PDF methodology was extensively applied to study nanostructured materials using short wavelength (epithermal) neutrons and high energy X-rays (Egami \& Billinge, 2003; Billinge \& Kanatzidis, 2004; Billinge 2008; Young \& Goodwin, 2011). PDF analyses were successfully applied to molecular compounds (Tucker et al., 2007, Rademacher et al, 2012), including $\mathrm{C}_{60}$ (Egami, and Billinge 2003), pharmaceutical materials (Moore et al., 2009, Billinge et al., 2010, Dykne et al. 2011), organic pigments (Schmidt et al., 2009, Schmidt, 2010), polymers (Petkov et al., 2005a, 2005b), organometallic compounds (Petkov \& Billinge, 2002) and metal-organic complexes (Wolf et al., 2012).The powder diagrams for PDF analysis are usually recorded with X-ray synchrotron sources. They may also be obtained from neutron spallation sources or from laboratory X-ray diffractometers. For high quality PDFs a short X-ray wavelength is preferred, e.g. Mo-K $\alpha_{1}$, Ag-K $\alpha_{1}$ or synchrotron radiation (e.g., see Dykne et al., 2011).

PDF curves can also be obtained using electrons. In transmission electron microscopes (TEMs) an operator can easily change the diffraction camera length thus setting various $Q$ ranges and electron wavelengths if necessary. Additionally, the operator can switch between imaging and diffraction mode, and select from which area of the sample the diffraction pattern should be recorded. The interaction of electrons with matter is much stronger than the interaction of X-rays or neutrons, and the electron beam can easily be focussed on a small spot, hence the required sample size is much smaller than for X-rays or neutrons. All these possibilities make PDF analysis from electron diffraction data an attractive alternative to Xrays or neutrons.

Electron diffraction (ED) has been long used for structure characterisation of single organic nanocrystals (Vainshtein, 1964, Dorset 1995). Due to the significant contribution of multiple scattering, ED was rarely used for an ab-initio structure analysis, mainly supporting structure analysis based on a combination of other structural methods: X-ray powder diffraction (Gorelik et al., 2010), NMR (Lotsch et al., 2007) or computational techniques (Voigt-Martin, 1995). Recently, with the development of 3D electron diffraction techniques (Kolb et al., 2007, Kolb et al., 2008), ab-initio structure analysis of organic materials became possible (Gorelik et al., 2009, Kolb et al., 2010; Gorelik et al., 2012). 
Powder electron diffraction (resulting in ring patterns) is usually used for structural fingerprinting (Làbàr, 2004; Moeck \& Rouvimov, 2009). The intensity variations within the rings may also be used for texture analysis of nanocrystals (Gemmi et al., 2011), but usually the rings are azimuthally integrated into $1 \mathrm{D}$ diffraction profiles. Obtaining quantitatively reliable powder diffraction intensities from electron microscopes is very rare, with only a few examples of a quantitative structural analysis of powder electron diffraction data in the literature, all from inorganic compounds (Weirich et al., 2000, Kim et al., 2009, Luo et al., 2011). There are a number of reasons, including the strong tendency for electrons to diffract dynamically, the difficulty of obtaining good powder averages from such small volumes of material, and the propensity for the electron beam to damage the sample. Rietveld refinement of organic compounds from powder electron diffraction data has never been done so far.

PDF studies have been made using electron diffraction data of inorganic amorphous materials and very thin polycrystalline inorganic films (Moss \& Graczyk 1969, Sproul et al. 1986, Anstis et al. 1988, McCulloch et al. 1999, Noerenberg et al. 1999, Hirotsu et al. 2003, Ankele et al. 2005, Hirata et al. 2006, Cockayne 2007, Hirata et al. 2007, Abeykoon et al. 2012), and more recently on inorganic nanoparticles (Farrow et al. 2010, Abeykoon et al. 2012). Here, for the first time we extend these studies to the electron PDF study of organic samples. The ways to overcome the instability of the samples in the electron beam is a major consideration for this work. Here we describe the experimental methods and data analysis approaches that yield electron PDFs (ePDFs) from organic solids, collected over a wide range of reciprocal space as required for quantitative or semiquantitative PDF work.

The molecular schemes of the materials we studied are shown in Figure 1.
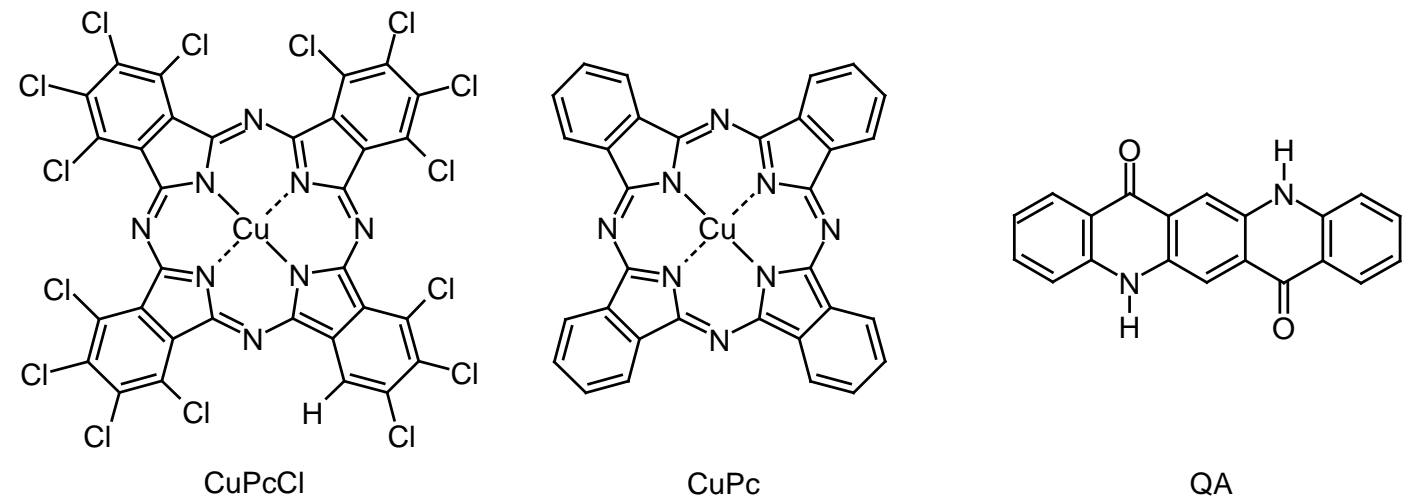

Figure 1

Chemical drawing of chlorinated copper-phthalocyanine (CuPcCl), copper-phthalocyanine (CuPc) and quinacridone (QA). CuPcCl is actually a mixture of isomers and molecules with different degrees of chlorination, with about $15 \mathrm{Cl}$ atoms per molecule in average. 
Chlorinated copper-phthalocyanine (in the following abbreviated as $\mathrm{CuPcCl}$ ) has the chemical composition $\mathrm{CuC}_{32} \mathrm{~N}_{8} \mathrm{Cl}_{\mathrm{x}} \mathrm{H}_{16-\mathrm{x}}$ with $\mathrm{x}$ about 15 , and is registered in the International Colour Index as C.I. Pigment Green 7. The advantage of this material is that is electron beamstable which may be used to validate our methods in the absence of the uncertainty of the beam damage issue. As an example for a beam-sensitive material, a situation more commonly found for organics, we chose the parent compound, copper phthalocyanine (CuPc, $\mathrm{CuC}_{32} \mathrm{~N}_{8} \mathrm{H}_{16}$ ) in its $\beta$-modification ( $\beta$-CuPc, C.I. Pigment Blue 15:3). The third example is a purely organic compound, quinacridone (QA, $\mathrm{C}_{20} \mathrm{H}_{12} \mathrm{~N}_{2} \mathrm{O}_{2}$, C.I. Pigment Violet 19) in its $\gamma$ modification ( $\gamma$-QA).

$\mathrm{CuPcCl}$ shows a deep green shade, $\beta$-CuPc is a standard blue pigment, and $\gamma$-quinacridone exhibits a bluish red shade. All three compounds are industrially produced on a 10,000 ton scale and used as pigments for colouring lacquers, coatings, plastics, printing inks and artists' colours (Herbst \& Hunger, 2004). The compounds are organic semiconductors and are used in optoelectronic devices such as organic LEDs or photovoltaic systems. The crystal structures of $\beta$-CuPc and $\gamma$-QA are known from X-ray single-crystal structure analyses (Brown, 1968; Mizuguchi et al., 2002; Paulus et al., 2007).

\section{Methods}

\subsection{Materials}

CuPcCl ( ${ }^{\circledR}$ Hostaperm Green GNX), $\beta$-CuPc $\left({ }^{\circledR}\right.$ Hostaperm Blue B2G) and $\gamma$-QA $\left({ }^{\circledR}\right.$ Hostaperm Red E3B) were obtained from Clariant GmbH, Frankfurt. All three samples used were industrially produced nanocrystalline powders. Since ePDF generally lends its use for the analyses of nanocrystalline materials, we used the materials as received and did not make any attempt to improve the crystallinity of the samples.

\subsection{Sample preparation for TEM}

Samples for TEM investigations were prepared by suspending the powders in n-hexane in an ultrasonic bath. A drop of the suspension was then placed onto a holey-carbon coated copper grid and dried in air. 


\subsection{Electron data collection}

TEM investigations were performed with a TECNAI F30 transmission electron microscope equipped with a field-emission gun operating at $300 \mathrm{kV}$ and an STEM unit. The diffraction data were recorded on a 1k GATAN CCD camera. Diffraction patterns were collected with a camera length of $380 \mathrm{~mm}$. In order to increase the Q-range of the data, the central (transmitted) beam was kept at a corner of the CCD area.

\subsubsection{Electron beam damage}

Inelastic scattering deposits energy into the material, which is then released by different means - either transformed into thermal vibration of the molecules, or into excitation of individual molecules which eventually can cause ionization and reorganization of molecular structure (e.g. bond cleavage and formation of cross-linked aggregates). Traditionally in electron crystallography the beam damage is defined as loss of crystallinity of the material as a result of electron irradiation (Kolb et al, 2010). As beam damage can cause reorganization of the molecular structure, obviously it is also a serious concern for poorly crystalline and amorphous organic materials.

The collective damage effects are usually quantified using the deterioration of the crystalline lattice, which is only possible for materials showing distinct Bragg peaks in diffraction patterns. It is unclear if the estimates of the critical electron dose for crystalline materials can be directly transformed to the amorphous state. The PDF analysis, being sensitive to the configuration of the near neighbor atomic configuration, should be the ultimate tool to study the collective radiation damage effects in organic materials and should be explored in the future.

There are several possibilities to improve the stability of organic material under electron radiation - cooling, enhancing the charge and heat transfer, but the most fundamental solution is a significant decrease of the intensity of the incident electron beam. The counteracting force to the decreasing of the illumination level is the reduction of the count statistics on the detector. Contemporarily most of the TEM data is recorded onto CCD cameras or image plates. Recently detectors built on CMOS technology for TEM appeared on the marked having superior sensitivity and therefore high potential for low-illumination level TEM investigations including diffraction data acquisition for beam-sensitive materials.

In this work three nanocrystalline organic samples were used. Electron diffraction patterns of all these materials showed Bragg-like peaks indicating that they have some crystallinity 
and are not truly amorphous. As an initial check for beam damage a series of diffraction patterns were collected from the same position on the samples showing a continuous decay of the reflection intensities. From the intensity decay plots a critical electron dose was estimated (Kolb et al, 2010). During the diffraction data collection for the PDF analysis electron dose rates were used causing a less than 5\% decay of the Bragg intensities (exact values are given below separately for each experimental setup). Setting up the illumination conditions far below the critical electron dose is essential for ensuring that the resulting PDFs are a good measure of the original structure of the materials under study.

\subsubsection{Selected Area Electron Diffraction (SAED) vs. nanodiffraction}

In selected area electron diffraction (SAED) the sample is illuminated with a parallel beam having a certain diameter $\mathrm{D}_{0}$. A selected area aperture is inserted at a plane conjugated with the specimen plane (below the specimen) with an effective diameter of $\mathrm{D}_{\mathrm{SA}}$. In order to keep the incident beam parallel and to have well defined diffraction geometry $\mathrm{D}_{0}$ is usually kept large, while $\mathrm{D}_{\mathrm{SA}}$ is selected according to the size of the specimen features (for instance, crystal size). In this geometry, the illuminated (and therefore subject to eventual beam damage) sample area is rather large $\left(\mathrm{D}_{0}\right)$, while the area effectively used for diffraction data collection - $\mathrm{D}_{\mathrm{SA}^{-}}$is just a small fraction of it. Nevertheless, when $\mathrm{D}_{0}$ is known a new diffraction pattern may be collected from a fresh (unexposed area) using a stage-shift of greater than $\mathrm{D}_{0}$. This classical SAED geometry can be used when diffraction lens settings cannot be changed and the sample features are relatively small. Typically, for beam sensitive materials the SAED geometry is modified: the diameter of the illuminated area $\mathrm{D}_{0}$ is reduced by focussing the incident beam. In the optimal case, the selected area for the diffraction information is collected from the same area that is illuminated $\left(D_{0}=D_{S A}\right)$. For these conditions there is no need to keep the selected area aperture in place. Combining different condenser lens settings and condenser aperture size one can obtain any beam size at the specimen principally down to a few nanometers; therefore this diffraction geometry is called nanodiffraction (though in order to collect diffraction patterns of poorly crystalline materials with good statistics and a reasonable powder average the actual area may be hundreds of nanometers or more).

A key step in the analysis is to convert the diffraction image from detector pixels to $\mathrm{Q}$. To do this the electron wavelength and effective camera length must be known. Typically the electron diffraction camera length is calibrated using a known standard material at specified illumination conditions (state of the condenser lens current). For these illumination conditions 
the diffraction patterns have to be focused using the diffraction lens. Selecting the illuminated area for nanodiffraction implies free modification of the illumination conditions and thus the convergence of the beam. As a result, the corresponding diffraction pattern is acquired at nonstandard lens settings, and appears defocused. Additional focussing of the diffraction pattern causes rotation and contraction/expansion of the diffraction pattern. The rotation is not relevant for powder (ring) patterns, but the contraction/expansion changes the effective camera length and therefore has to be taken into account.

A way to compensate for the additional focussing was proposed by Kolb et al. (2011): for a known standard material a set of diffraction patterns is collected using diverse beam convergence. Each pattern is focused using the diffraction lens. Then, the interplanar distances seen on the patterns are measured and the effective camera length is calculated. This effective camera length shows a linear trend when plotted against the diffraction lens current value. A linear fit to the measured data can be used as a calibration curve for any nanodiffraction pattern. The final accuracy of $d_{\text {hkl }}$ determination using this procedure has statistical character and is typically below $2 \%$.

\subsubsection{Data collection using TEM and nanodiffraction}

The data were acquired at a medium magnification of 15,000x in TEM mode. The beam size was set to $1 \mu \mathrm{m}$ diameter (Figure 2a). After the necessary alignments were done, including the eucentric height adjustment and beam size selection, a reasonably low illumination level of the beam has to be selected. The intensity of the beam is dictated by the radiation stability of the sample. Ideally, this characteristic electron dose that can be tolerated by the sample should be estimated as described above (Kolb et al., 2010) before the diffraction data collection for PDF analysis. Following this procedure we determined that the electron dose rate during measurements for $\mathrm{CuPcCl}$ be kept at the level of $15 \mathrm{e}^{-} / \AA^{2} \mathrm{~s}$, and for $\beta$-CuPc and $\gamma$-QA at $0.7 \mathrm{e}^{-} / \AA^{2}$ s. A slight convergence of the incident beam required additional focussing of the diffraction pattern. The effective diffraction camera length was calibrated using the calibration plot and was $415 \mathrm{~mm}$ (nominal $380 \mathrm{~mm}$ ).

The stage was shifted mechanically in a grid in steps of $1 \mu \mathrm{m}$. At each stage position first an electron diffraction pattern was recorded with $1 \mathrm{~s}$ exposure time, then the image of the area was taken. The procedure of blind recording of diffraction patterns mimics the low dose acquisition technique used in biological studies (Dubochet et al., 1988). The procedure can easily be automated (Zhang, et al., 2003) and deliver high quality data in a few minutes. 
There are different possibilities to decrease the intensity of the incident electron beam for a FEG source. For FEI TECNAI TEMs several parameters controlling the beam are available gun lens, spot size and the extraction voltage. A proper combination of these parameters can create a low-intensity electron beam suitable for analysis of organic and biological objects. In order to measure the electron dose on the sample, first the electric current on the scintillator screen is measured. TECNAI TEMs offer a possibility to measure the current on the screen within a Low Dose unit (mainly used for the biological applications). The electric current can then be correlated with the counts on the camera, giving a conversion rate of the camera how many counts correspond to one primary electron. Knowing the conversion rate, the electron dose can be calculated from an image recorded onto a camera with a known exposure time.

In total, 50 pairs of diffraction/image pairs were collected from each sample. This approach not only maximises the counting statistics whilst minimizing beam damage, but increases the powder statistics (the number of crystallites that are averaged over to obtain the integrated diffraction patter) in a natural way. Based on the corresponding images these pairs could be classified into: (i) diffraction patterns from the material, (ii) diffraction patterns from the carbon film only, and (iii) diffraction patterns including copper grid parts. The patterns from the carbon film were averaged and used for background estimation; the patterns with copper reflections were discarded. All diffraction patterns from the material were summed together, and likewise for all the background diffraction patterns, and these integrated images were used for further processing.

\subsubsection{STEM / nanodiffraction}

A particularly soft illumination setup can be realized by combining scanning transmission electron microscopy (STEM) with nanodiffraction mode (Kolb et al., 2007). STEM with high-angle annular dark field (HAADF) detectors allow one to obtain very high contrast images of the sample while keeping the effective electron dose low. Electron diffraction patterns in nanodiffraction mode can then be recorded from certain areas of the sample seen in the STEM image. This method is particularly beneficial for non-homogeneous samples.

An automated acquisition module developed for a different application, automated diffraction tomography - ADT (Kolb et al., 2007; Kolb et al., 2008), provides the possibility to scan a certain area selected in a STEM image in diffraction mode. We used this module here to obtain data for our PDF study. The size of the scanned area was set to a square with a side length of $1 \mu \mathrm{m}$, and the beam size for nanodiffraction was kept at $100 \mathrm{~nm}$ (Figure $2 \mathrm{~b}$ ). 
Thus 10 x 10 electron diffraction patterns could be collected without overlap in a $1 \mu \mathrm{m}$ square. The electron dose rate for $\mathrm{CuPcCl}$ was $10 \mathrm{e}^{-} / \mathrm{A}^{2} \mathrm{~s}$, for $\beta$-CuPc and $\gamma$-QA at $0.3 \mathrm{e}^{-} / \mathrm{A}^{2} \mathrm{~s}$, which is lower than the doses rates for TEM mode. As for the data collection in TEM mode, the exposure for a single diffraction pattern was $1 \mathrm{~s}$. Diffraction patterns for the background estimation were collected from an adjacent area that included no particles.
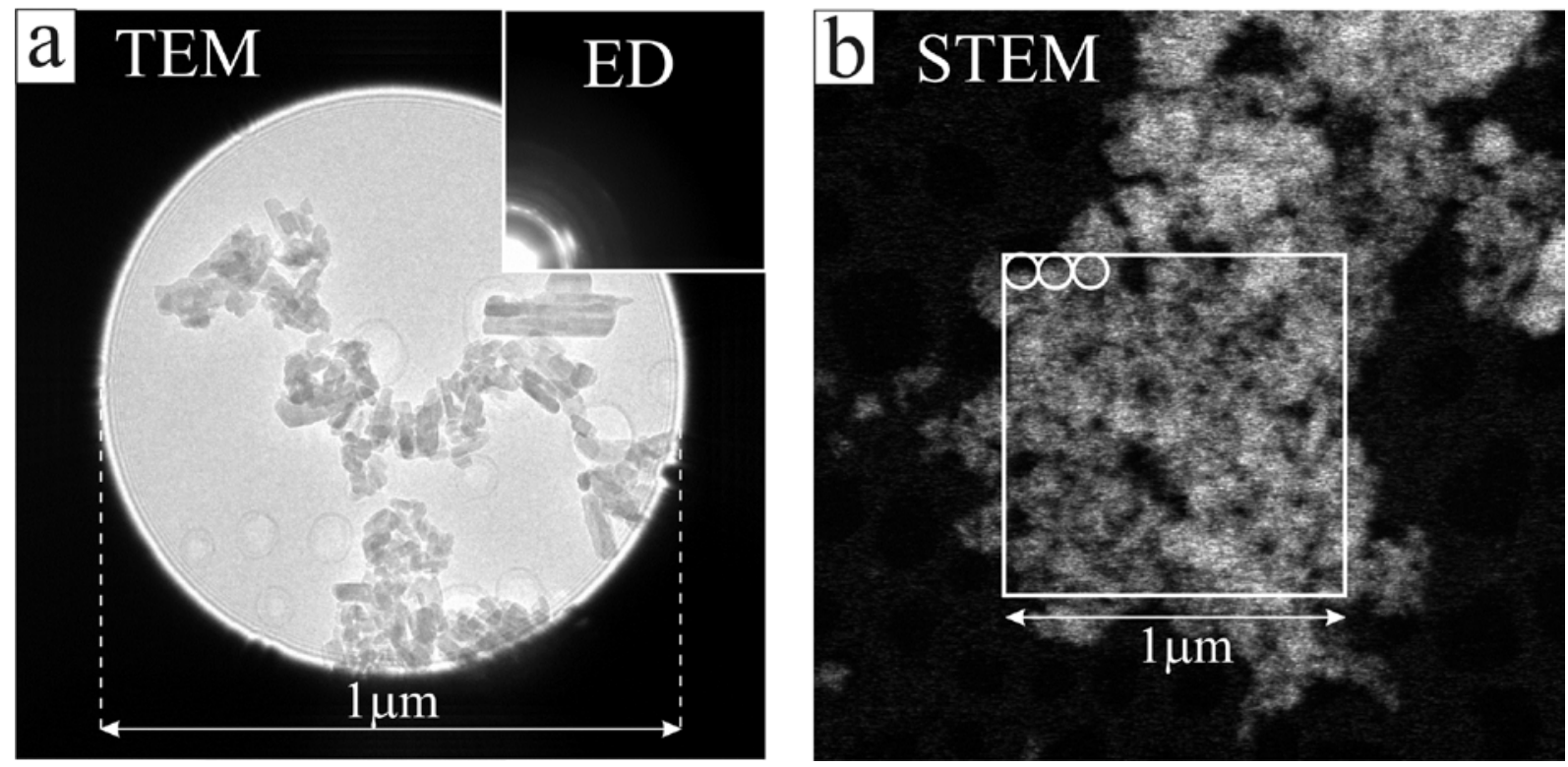

Figure 2

Different experimental setup for electron diffraction data acquisition ( $\mathrm{CuPcCl})$ : a) in TEM mode using either SAED with the beam size equal to the size of the SA aperture, or nanodiffraction with the same beam size (the area is scanned using stage shift), insert: an averaged electron diffraction pattern centred in the corner of a CCD area; b) in STEM mode using nanodiffraction with the beam size not causing overlapping of diffraction positions (the area is scanned using beam shift).

\subsubsection{Data reduction for electron diffraction}

As already mentioned above, the diffraction patterns were recorded with the central beam shifted to a corner of the detector. The centring of the patterns was made by analysing the gradient of the central beam as described in Kolb et al., (2007). After the patterns were centred, several patterns from different parts of the sample, as described above, were averaged in order to increase the signal to noise ratio due to the counting statistics and to improve the powder average. Averaged patterns were then azimuthally integrated and normalized by a number of 2D pixels fallen into each bin of constant Q-value. The integration procedure was done by a home-written program and produced integrated intensity vs. Q. Elliptical distortion (Capitani, et al., 2006; Lábár 2008; Lábár 2009; Mugnaioli, et al., 2009), often observed in electron diffraction patterns (typically below 2\%) was neglected. The same procedure was 
applied for diffraction patterns recorded from the supporting holey-carbon film for background estimation.

\subsection{X-ray diffraction}

For comparison with the electron diffraction patterns, X-ray powder diffraction data of all samples were recorded. The samples were measured in transmission geometry using STOE Stadi-P diffractometers equipped with Ge(111) monochromators. The samples were contained in sealed glass capillaries which were rotating during the measurements to increase powder averaging. Measurements were performed with Mo-K $\alpha_{1}$-radiation $(\lambda=0.7093 \AA)$. We used an image-plate position-sensitive detector (IP-PSD) with a $2 \theta$ range of $2-140^{\circ}$, which corresponds to a $\mathrm{Q}_{\max }$ of $16.6 \AA^{-1}$. The background was determined by measuring an empty capillary under identical experimental conditions.

\subsection{Obtaining the PDF}

The PDF is obtained from the integrated 1D electron diffraction patterns using a homewritten program PDFgetE. This program subtracts the background intensity and makes corrections for intensity aberrations in the data such as coming from sample absorption, incoherent multiple scattering and inelastic scattering (Abeykoon, 2012). It normalizes the data by the average electron form factor of the sample and for the incident flux to obtain the properly normalized structure function, $S(Q)$ and then $F(Q)$. Finally it does the Fourier transform in Equation 1 to obtain the PDF. The background intensity can be scaled and $\mathrm{Q}_{\max }$, the maximum value of $\mathrm{Q}$ over which data are used in the Fourier transform, can be varied by the user in such a way as to obtain the best possible PDF given the data. A public release of the program is planned for the future.

\subsection{Modelling}

PDFs can be calculated from structural models (Egami and Billinge 2003) and it is common to optimize model parameters by updating them in such a way as to get a good fit of a calculated PDF to a measured one. A widely used program for doing this is PDFgui (Farrow et al., 2007). For the $\beta$-CuPc sample we used PDFgui to calculate the PDF from single-crystal data from the literature (Brown, 1968). PDFgui is not adapted for refining structures from molecular systems and so we did not carry out structure refinements. However, certain profile parameters were adjusted to improve the agreement. These were scale-factor, $Q_{\text {damp }}$ (which 
accounts for the effects of the finite resolution of the measurement) and $S_{\text {rat }}$, a factor that sharpens PDF peaks at low-r below a cutoff, $\mathrm{r}_{\text {cut }}$. This mimics the effects seen in molecular systems that PDF peaks from intra-molecular atomic pairs tend to be much sharper than the peaks from the inter-molecular correlations.

\section{Results and discussion}

In order to differentiate between the different radiation sources, we introduce the names 'ePDF' for PDF from electron diffraction data ('xPDF': from X-ray data, 'nPDF': from neutron data) and 'eF(Q)' for $F(Q)$ from electron diffraction data ('xF(Q)': from X-ray data, 'nF(Q)': from neutron data).

\subsection{Chlorinated copper-phthalocyanine (CuPcCl, Pigment Green 7)}

Figure shows the averaged electron diffraction patterns from $\mathrm{CuPcCl}$ obtained using the TEM / nanodiffraction mode. The direct beam is in the lower left hand corner of the image. Debye-Scherrer powder diffraction rings are clearly evident in the raw data (panel a), though there is some distortion apparent along a radial direction at around $30^{\circ}$ to the vertical. This aberration is a ghost image of the electron source and it appears also in the background pattern shown in Figure b. Figure 3c shows the data of Figure 3a after subtracting the background. There is a small intensity modulation along the rings indicating an imperfect powder average, which is mitigated somewhat after the azimuthal integration into a $1 \mathrm{D}$ profile.
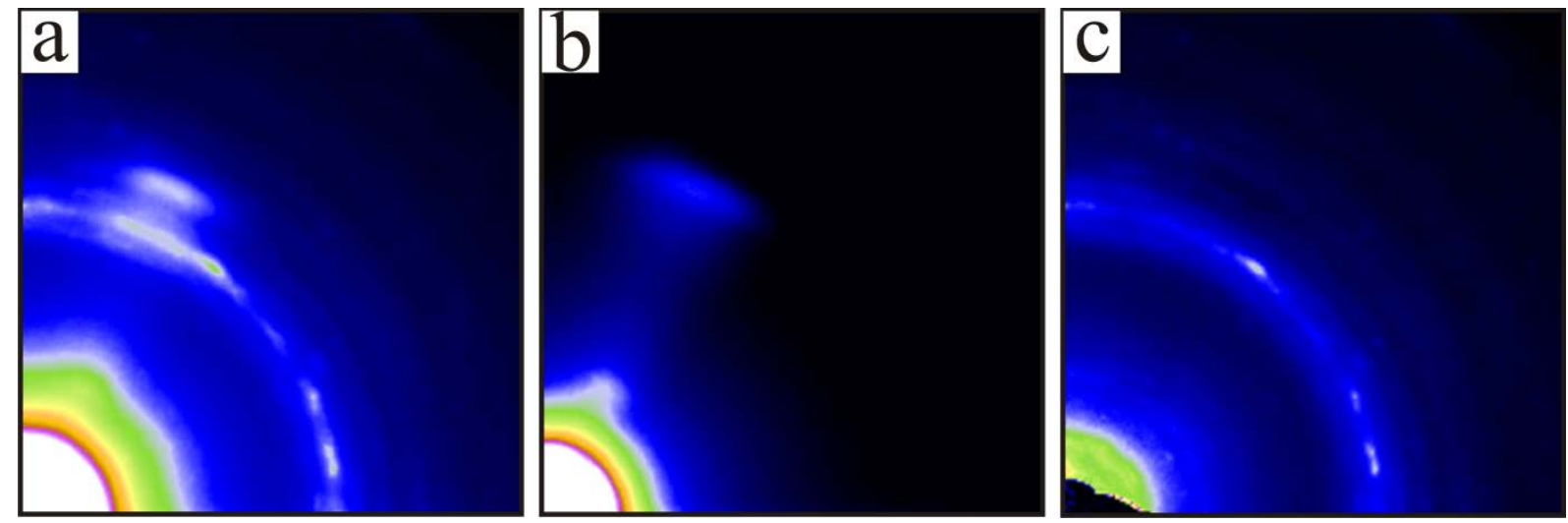

Figure 3

False-colour images of the electron diffraction patterns on the CCD detector from the CuPcCl sample. (a) a diffraction pattern after summing multiple exposures, as described in the Methods section. Notice the pattern is not circularly symmetric because of an image of the electron source. (b) Background image 
from regions away from the $\mathrm{CuPcCl}$ material. (c) Subtraction of the background image from the $\mathrm{CuPcCl}$ diffraction pattern showing the clean diffraction pattern.

The electron scattering data after the background subtraction and 1D integration steps are shown as the blue and green curves (collected in TEM and STEM mode correspondingly) in Figure 4a. In comparison, we also show data from the same sample collected using Mo X-rays (red curve in Figure 44a). Although the electron diffraction data has much lower Q-space resolution (about $13 \mathrm{~A}^{-1}$ ) than the $\mathrm{X}$-ray data, the same overall features are evident, though significantly broadened in the electron diffraction data. This is usually a good trade-off for PDF work, where currently the method of choice for X-ray PDF measurements is the low resolution but high throughput rapid acquisition PDF (RAPDF) mode (Chupas et al. 2003, Billinge et al., 2010).

a)

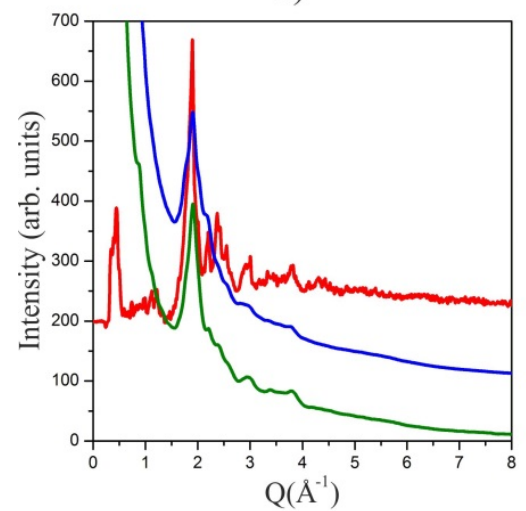

b)

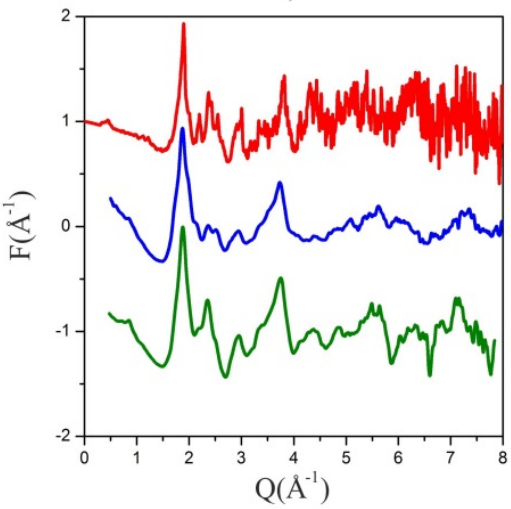

- Mo X-ray data

- electron data (TEM mode)

- electron data (STEM mode) c)

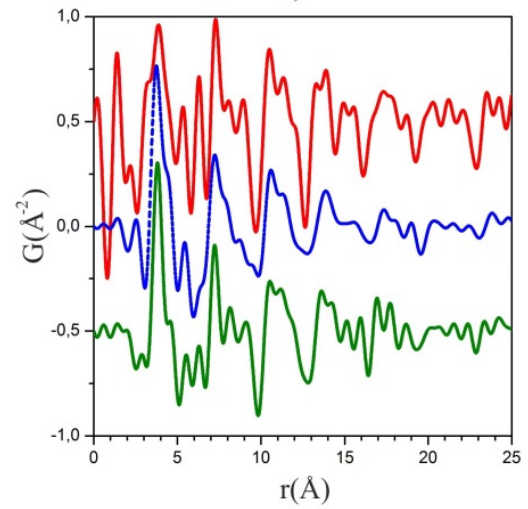

Figure 4

Comparison of electron diffraction and X-ray diffraction data of $\mathrm{CuPcCl}$. (a) the red curve shows the Xray data collected with Mo-K $\alpha_{1}$ radiation, the blue and green curves show the electron diffraction data collected using the TEM / nanodiffraction approach (presented in Figure 3c) and STEM / nanodiffraction mode after azimuthal integration and with the $x$-axis converted to $Q$, resp. (b) red, blue and green curves: the same data as in (a) converted to the reduced structure functions, $F(Q)$. (c) the PDFs obtained from the $F(Q)$ functions in the (b) pane, following the same color scheme.

When the data in Figure 44a are processed, the curves $F(Q)$ presented in Figure 4b are produced. Despite being measured for much less time (1 second exposure time per diffraction pattern, in this data-set 37 patterns were averaged) the electron data have sufficient statistics in the high-Q region, as shown by the blue and green curves, which shows TEM / nanodiffraction data. The bottom pale blue curve is another $\mathrm{eF}(\mathrm{Q})$ which was obtained using the STEM / nanodiffraction approach. Again, the positions and relative amplitudes of the 
features are well reproduced, and in this mode we see that the Q-space resolution of the measurement is in fact a little higher than for the TEM / nanodiffraction data. Clearly, both TEM and STEM nanodiffraction produce $F(Q)$ functions that strongly resemble those from the x-ray data, once the measurement at a much lower Q-space resolution is taken into account.

Finally, in Figure 44c, the PDFs from the data-sets shown in Figure 4b are plotted. A direct comparison can be made between the ePDF curves (in blue and green) and the Mo-K $\alpha_{1}$ xPDF curve (in red) as they were processed with the same $\mathrm{Q}_{\max }$ of $8 \mathrm{~A}^{-1}$. The curves have been scaled to emphasize the overlap in the high-r region. This scaling means the low-r peaks have different amplitudes between the ePDF and xPDFs. This results from a combination of the different resolution of the X-ray and electron diffraction data, and may possibly be in part due to some dynamical scattering effects which tend to sharpen the low-r peaks in the PDF (Abeykoon et al, 2012). The peaks that are most affected are the sharp intra-molecular atomatom peaks that originate from the covalently bonded atoms on rigid subunits of the molecule. These peaks are mostly affected by the electron beam induced damage inducing covalent bond cleavage and slight atomic displacement. The information contained in these peaks is often of less interest in the study of organics. Advanced modelling codes that account for this low-r sharpening in molecular PDFs are under development and these may be extended to correct for these aberrations. Of greater interest is the high degree of similarity in the high-r region from an experiment that is much quicker and often more accessible since powder diffractometers with Mo sources are rare in laboratories.

This clearly demonstrates the potential of the ePDF method for producing PDFs that are suitable for a quantitative or semi-quantitative analysis using modelling. The exact level of accuracy of structural parameters refined from the data remains to be established. Some parameters should be very robust, such as nanoparticle lattice parameters. Parameters that depend sensitively on the amplitudes of the PDF peaks, and in particular on the r-dependence of the amplitudes, are more susceptible to the effects of dynamical scattering, and this needs to be studied more systematically in the future. However, these results show that ePDFs can be obtained from organic materials using diffraction data aquisition in TEM or STEM mode, that are highly similar to those obtained using x-ray methods. 


\subsection{Copper-phthalocyanine ( $\beta$-CuPc, Pigment Blue 15:3)}

The results for the $\beta$-CuPc compound are shown in Figure 5. The experiments and data analysis were more difficult in this case because of the beam sensitivity of the sample. The background-subtracted diffraction pattern taken in the TEM / nanodiffraction mode is shown as the inset. The powder rings are smooth although a little spotty, indicating that there is some crystallinity in the sample. Figure 5a shows the data after $1 \mathrm{D}$ integration and converting to $F(Q)$. Again, the $\mathrm{eF}(\mathrm{Q})$ (blue curve) and the $\mathrm{xF}(\mathrm{Q})$ (red curve) from the laboratory X-rays are shown for comparison. The main features of the X-ray curve are reproduced in the eF(Q). The electron data are shown below in blue extended all the way to the maximum Q-range measured of $7.7 \AA^{-1}$; features in the data are clearly evident all the way out, measured with good statistics. This presents us with the opportunity to obtain a PDF with good real-space resolution by Fourier transforming over this range.

a)

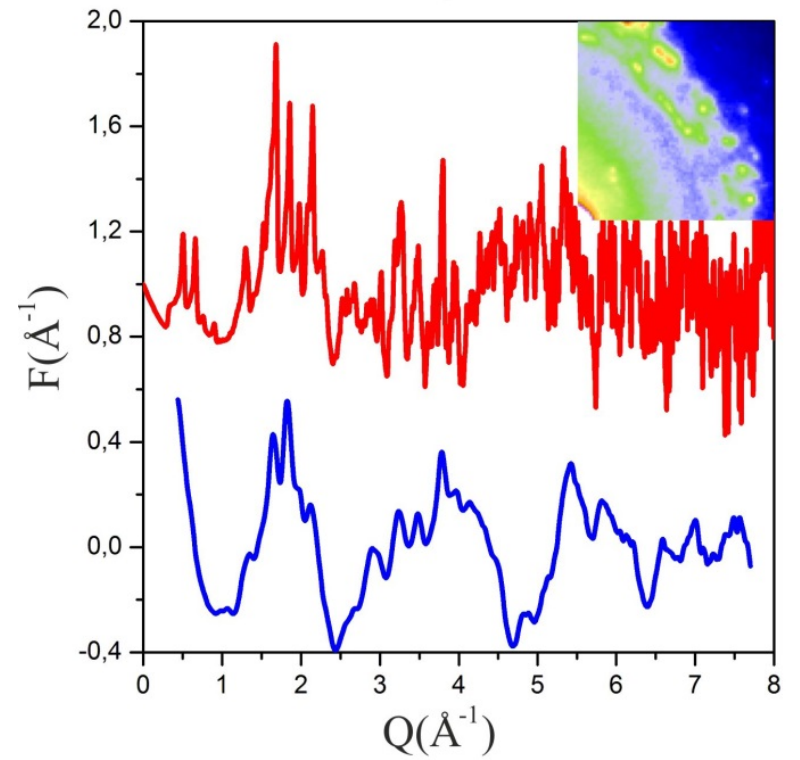

b)

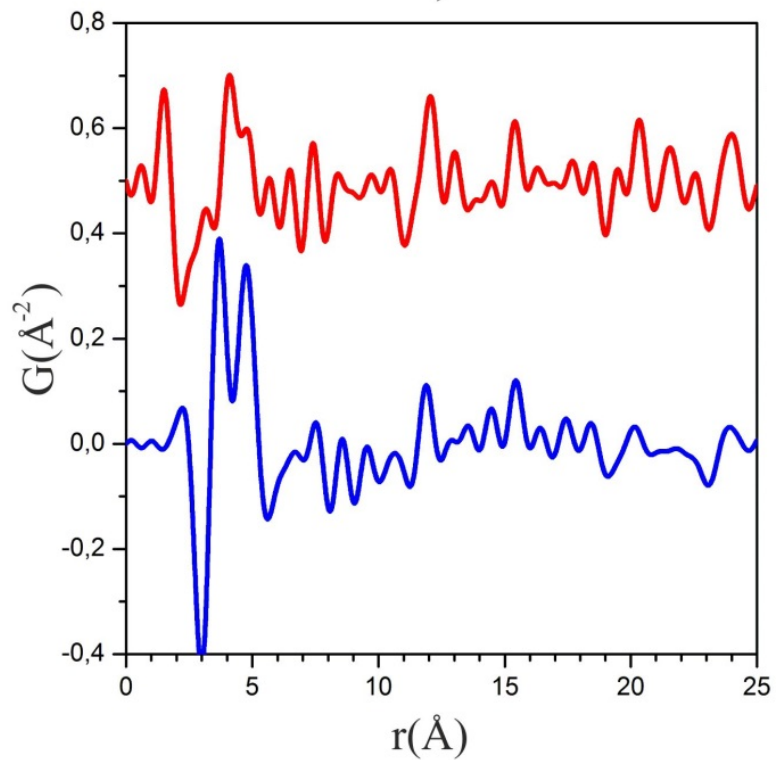

Mo X-ray data

- electron data (TEM mode)

Figure 5

Comparison of electron and X-ray diffraction data for $\beta$-CuPc: (a) red curve:u Mo-Ka $\alpha_{1} \times F(Q)$, blue curve: the $F(Q)$ from electron diffraction data collected in TEM / nanodiffraction mode. The inset shows the image of the background subtracted electron diffraction pattern before 1D integration. (b) The PDFs obtained from the $F(Q)$ functions in (a), following the same colour scheme. The ePDF curve has been scaled to emphasize the agreement in the high-r region.

The resulting PDFs are shown in Figure 5b. The features of the Mo-xPDF are reproduced in position and relative intensity by the ePDF, though as before we have scaled the ePDF to 
emphasize the good agreement in the high-r region. The ePDF curve drops faster in amplitude compared to the Mo-xPDF curve due to the lower Q-resolution in the electron diffraction data. As in the case of $\mathrm{CuPcCl}$, the most significant disagreement between the PDFs is observed in the low-r region, representing the short interatomic distanced within a molecule.

Figure 6 demonstrates a comparison between the best ePDF, obtained using a $\mathrm{Q}_{\max }$ of 7.7 $\mathrm{A}^{-1}$ from the TEM / nanodiffraction data and the PDF calculated from a model of the crystal structure determined from single-crystal data (Brown, 1968). The features of the curves are reproduced well. However, it should be noted that no true "fit" of the data varying the structural parameters has been done. The PDF was calculated from the model and parameters that account for scale-factor, $\mathrm{Q}_{\max }$ effect and resolution damping were applied to the calculated PDF to give a better overall agreement. The purpose of this exercise is not to obtain a quantitative fit to the data, which is not possible at the present time because of limitations of the fitting program, PDFgui, for fitting molecular solids, but to show that the ePDF obtained from the electron diffraction data is well reproduced by a PDF calculated from the known structure of the material. Quantitative fitting of the PDFs from molecular solids will be the subject of future studies. However, despite the challenges of studying organic nanometerials using ePDF approaches, these early results show great promise.

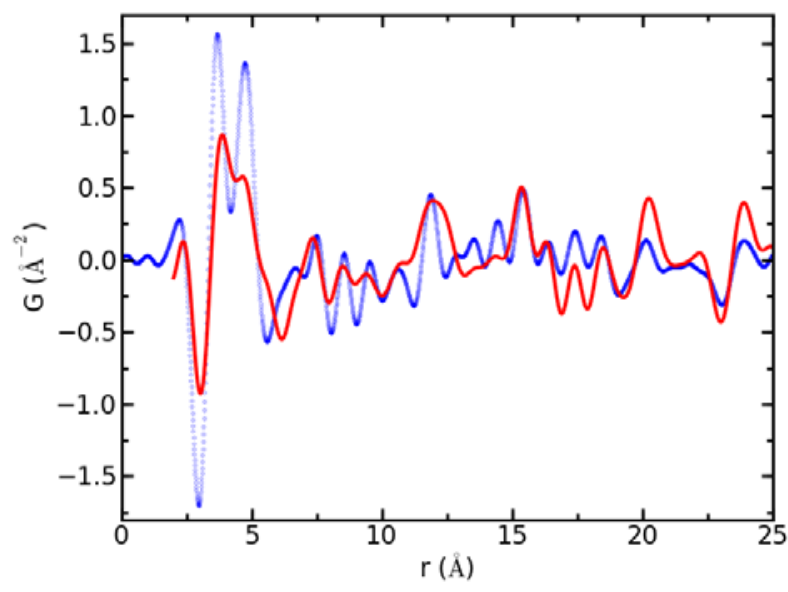

Figure 6

Comparison of the ePDF with a simulated PDF from the known crystal structure: blue dotted line: ePDF shown in Figure $5 b$ calculated with $Q_{\max }=7.7 \mathrm{~A}^{-1}$; red line: simulated PDF from the structural model of $\beta$ CuPc (Brown, 1968). 


\subsection{Quinacridone ( $\gamma$-QA, Pigment Violet 19)}

Quinacridone is an example of a purely organic compound that is also beam-sensitive. We demonstrate that our approach yields ePDFs that are of comparable quality as shown above for the $\beta$-CuPc sample. Averaged and background subtracted electron diffraction patterns of $\gamma$-QA were similar to those of $\beta$-CuPc - spotty powder rings. Figure 7 shows the eF(Q) functions from TEM / nanodiffraction data (blue curve in the panel a) plotted together with the X-ray F(Q) (red profile in Figure 7a) and, apart from the lower resolution of the electron data there is a good reproducibility. This is also evident when the data are Fourier transformed to the PDFs (Figure 7b). This example shows that ePDF is possible for purely organic compounds, too.

a)

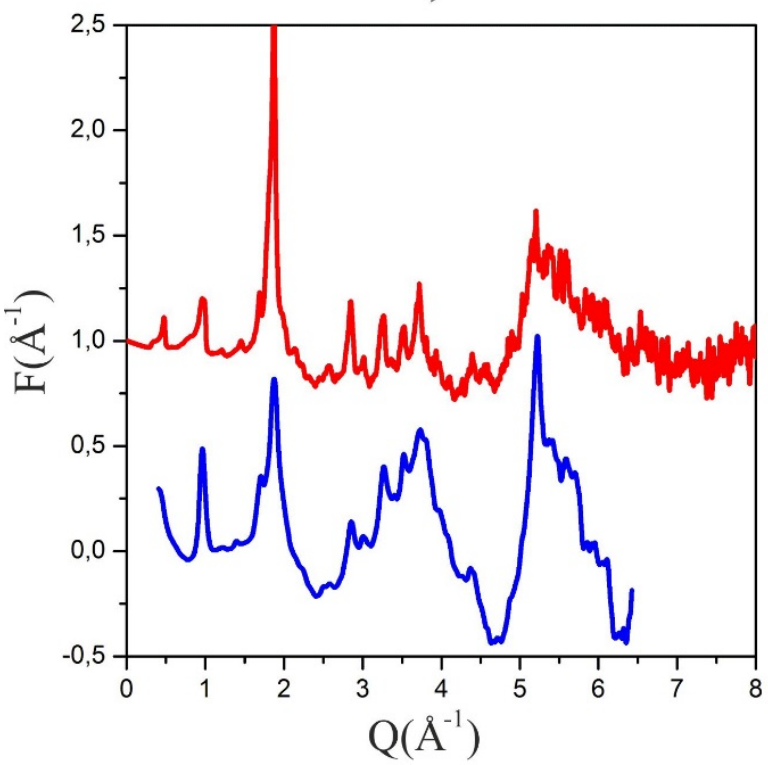

b)

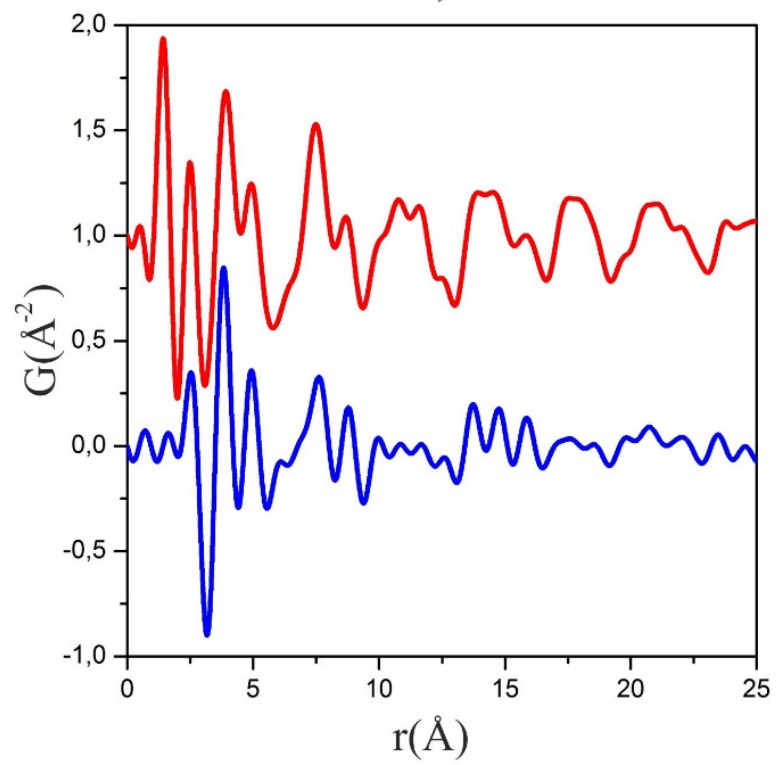

Mo X-ray data

- electron data (TEM mode)

Figure 7

Comparison of electron and $X$-ray diffraction data for $\gamma$-QA: (a) red curve: $x F(Q)$, blue curve: the $F(Q)$ from electron diffraction data collected in TEM / nanodiffraction mode. (b) The PDFs obtained from the $F(Q)$ functions in (a), following the same colour scheme. The ePDF has been scaled to emphasize the agreement in the high-r region. 


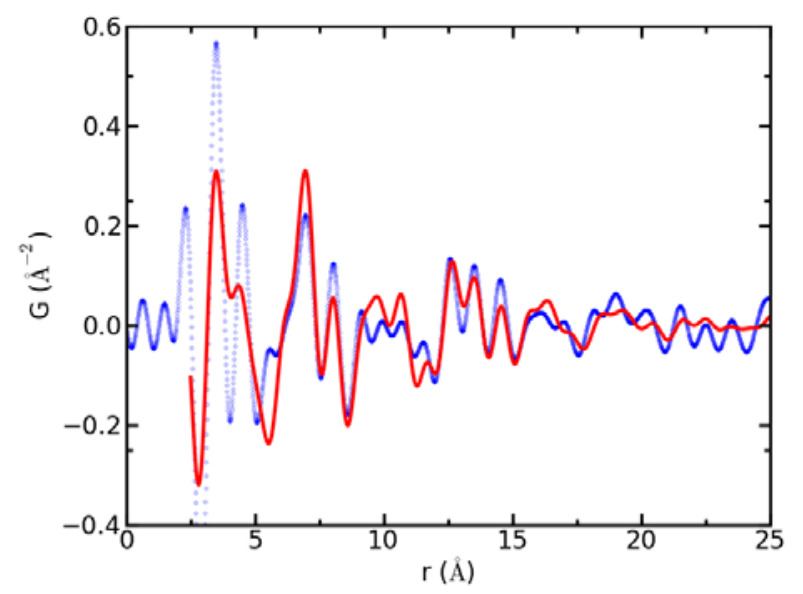

Figure 8

Comparison of the ePDF with a calculated PDF from the known crystal structure of $\gamma$-QA: blue dotted line: ePDF shown in Figure $7 \mathrm{~b}$ calculated with $Q_{\max }=7.0 \mathrm{~A}^{-1}$; red line: $P D F$ calculated from the structural model of $\gamma$-QA (Paulus et al., 1989, 2007).

As it was the case for $\beta$-CuPc we were able to compare a calculated PDF from the known crystal structure with the ePDF and this is shown in Figure 8. The structural model was taken from Paulus et al. (1989, 2007). Again, although the structure model is not being fitted to the data the features of the two curves are well reproduced giving further proof that the electron diffraction approach we have taken results in PDFs that will be suitable for modelling, when the required modelling codes become available.

\subsection{Comparison of PDF obtained from different methods for recording the electron diffraction data}

\subsubsection{General}

The best strategy in order to minimize electron beam induced damage effects to the structure is to acquire data from fresh unexposed area. This can either be achieved by a controlled stage shift or beam shift after each exposure. A mechanically controlled stage shift is more efficient for relatively large steps and therefore can be best used combined with a large illumination spot in TEM mode. The large beam size covers more particles and therefore increases the powder average of the diffraction data. SAED (selected area electron diffraction) using the SA (selected area) aperture limiting the area of interest can be used when a large beam size is necessary in order to keep proper geometry of the diffraction experiment - the incident beam stays parallel, and small features of the sample (included in the SA aperture) can be separated. Using this diffraction geometry one should keep in mind that the area not 
included in the SA aperture but illuminated during the exposure is not used for data collection. In the nanodiffraction geometry (SA aperture out) all illuminated area is used for the data collection. Electron diffraction data collected using the stage shift is easy to realise experimentally and generally should result in better statistically averaged diffraction data.

Beam shift can be used for shorter shift distances. It can be most efficiently combined with STEM imaging mode. STEM / nanodiffraction combination is a little more sophisticated experiment and requires a dedicated acquisition module controlling the beam shift. Since it averages the diffraction data over a smaller area, it has generally poorer statistics as the TEM / stage shift approach with the large beam size. Nevertheless, since the diffraction data is collected from known positions within the STEM image, non-uniform samples can be analysed.

\subsubsection{Comparison of different electron diffraction setups for determining the PDFs}

Our experiments indicate that it is much easier to get good statistics on the data, both counting statistics and powder average, using a large beam size realized in TEM / nanodiffraction mode, than fine scanning in STEM / nanodiffraction. The latter works too, as shown in the $\mathrm{CuPcCl}$, where high-quality PDFs were obtained in the STEM / nanodiffraction mode. However, significantly more averaging must be done in the STEM / nanodiffraction mode in order to get sufficiently well averaged data. This may still be attractive for nonuniform beam sensitive samples as particular area of the sample can be selected for sampling in STEM image. Furthermore, homogenous truly amorphous samples are isotropic and do not require much averaging. For most cases, the TEM / nanodiffraction or SAED mode is sufficient and more straightforward for obtaining high quality data for ePDFs.

Electron diffraction data for PDF analysis can also be collected using a non-TEM experimental setup. The idea of a dedicated camera for electron diffraction experiments in transmission mode was very popular in the middle of the last century (Coltman 1955, Vainshtein, 1964). Several cameras were built and used for structure analysis of polycrystalline materials. Unfortunately no commercial version of the electron diffraction camera appeared, which may change in the near future triggered by the last achievements of the electron crystallography. Electron diffraction data obtained in reflection geometry (Zewail, 2006) does not give information about the bulk, but carries the information the structure features of the near surface region (LEED, RHEED). Principally these kind of data can also be used for PDF analysis, analogous to grazing angle data collection in X-rays (Elschner et al., 2011). The PDF analyses using grazing incidence with electrons, also lower 
energy electrons (Farrow et al., 2010) has already been demonstrated for inorganic gold nanoparticles. Similar investigations should also be possible e.g. for organic nanoparticles or amorphous or nanocrystalline organic films.

\section{Conclusion}

Despite the challenges, we have shown that PDFs from nanocrystalline organic and metalorganic compounds can be determined from electron diffraction that reproduce well xPDFs from the same materials, and that are suitable for subsequent modelling. In terms of signal-tonoise, and in overall appearance, the PDFs are comparable in quality to those obtained from an X-ray setup, though for a fully quantitative analysis more work is required to validate which refined parameters are reliable and which are determined with less reliability due to dynamical scattering effects, for example. The data collection must be carried out carefully to ensure there is not significant beam-damage evident in the diffraction patterns, and also to ensure sufficient counting statistics and a good powder average. The resulting PDFs are good for finger-printing the nanocrystalline structure, and we show that they may be fit with the PDFs from structural models where they are available. Here we note that modelling codes for fitting the PDFs of molecular materials are under development in our group and will be available in the future, allowing for a proper validation of ePDF refined parameters.

The high Q-range accessible in principle in the TEM, and the sensitivity to light atoms, means that ePDF may become a powerful method for nanostructure characterization. The maximum values of $\mathrm{Q}$ obtained here were modest, and future work will also be invested in protocols for collecting data in TEMs over wider ranges of momentum transfer. Potential advantages of electron diffraction derived PDFs are that they can be obtained from small quantities of material, and material in special geometries, such as in the form of a thin film. Whilst they are not currently straightforward to obtain, this may be remedied in the future with special attachments to microscopes coupled with dedicated software for data collection and reduction.

Possible applications of ePDF over xPDF include:

1. Leveraging the small amount of sample materials required: 1ng to $1 \mathrm{pg}$ may be fully sufficient, which can be of importance especially for difficult to produce pharmaceutical or biological samples, for instance, nanoparticles produced by electrospraying (Marijnissen et al., 2010). A sample thickness of $10-100 \mathrm{~nm}$, preferably $30-100 \mathrm{~nm}$, is sufficient. 
2. Thin films with a thickness of about $10 \mathrm{~nm}$ to $1 \mu \mathrm{m}$ (for organics) can be investigated. Possible applications include organic films for optical or photovoltaic devices, OLEDs or organic transistors.

3. The electron microscope can be used to record images of the material. The electron beam can be focussed to small areas (e.g. $10 \mathrm{~nm}$ ). It is easily possible to select, which section of the sample should be investigated, which is useful especially for non-homogeneous samples, e.g. tablets consisting of a nanocrystalline or amorphous active pharmaceutical ingredient dispersed in an excipient, or a nanocrystalline organic pigment dispersed in a laquer, coating, polymer, paint or printing ink.

4. The element composition can simultaneously be determined by EELS or EDX analyses. EELS also allows to determine the hybridization state, e.g. of C atoms.

5. And: Transmission electron microscopes are cheaper and more widely spread than synchrotron or neutron sources.

This work shows that the investigation of local structures of organic and organo-metallic compounds is possible by means of PDF analysis from electron diffraction data. We have shown the application for organic pigments, but the method can also be applied to other materials like pharmaceuticals, compounds in organic optoelectronics (e.g. OLED and organic photovoltaics), biological samples, and to thin films of organic and organometallic compounds. There is a real possibility that, with improvements in modelling software targeted at molecular materials, and to include corrections for resolution and dynamical scattering effects in the data that have been identified in this study, PDFs with quantitative reliability approaching that from X-rays and neutrons will be possible. These developments are currently under way and will be reported in the future.

\section{Acknowledgement}

The authors thank Clariant GmbH (Frankfurt) for providing the industrial samples and $\mathrm{Dr}$ Lothar Fink and Edith Alig (Goethe University, Frankfurt) for X-ray powder diffraction measurements. Work in the Billinge group was supported by Laboratory Directed Research and Development (LDRD) Program 12-007 (Complex Modeling) at the Brookhaven National Laboratory. Finally, the authors would like to thank Sonderforschungsbereich 625 (Mainz) and DFG-Schwerpunktprogramm 1415 for financial support.

\section{References}


Abeykoon, M., Malliakas, C.D., Juhás, P., Božin, E.S., Kanatzidis, M.G., Billinge, S.J.L. (2012). Quantitative nanostructure characterization using atomic pair distribution functions obtained from laboratory electron microscopes. Z. Krist. 227, 248-256.

Ankele, J. E., Mayer, J., Lamparter, P., Steeb, S. (2005). Quantitative Electron Diffraction Data of Amorphous Materials. Z. Naturforsch. 60a, 459-468.

Anstis, G.R., Liu, Z., Lake, M. (1988). Investigation of amorphous materials by electron diffraction - the effects of multiple scattering. Ultramicroscopy, 26, 65-70.

Barnes, A. C., Fischer, H. E. Salmon, P. S. (2003). Neutron and X-ray diffractions for the structural study of liquids and glasses (in french). Journal de Physique IV, 103, 359-390.

Bates, S., Zografi, G., Engers, D., Morris, K., Crowley, K., Newman, A. (2006). Analysis of amorphous and nanocrystalline solids from their X-ray diffraction patterns. Pharm. Res. 23, 2333-2349.

Billinge, S. J. L. (2008a). Nanoscale structural order from the atomic pair distribution function (PDF): There's plenty of room in the middle. J. Solid State Chem. 181, 16981703.

Billinge,S. J. L. (2008b). Local Structure from total scattering and atomic Pair Distribution Function (PDF) analysis. In: Powder diffraction: theory and practice, (Royal Society of Chemistry, London England, 2008), R. E. Dinnebier and S. J. L. Billinge, Eds., pp. 464 493.

Billinge, S. J. J., Dykhne, T., Juhás, P., Božin, E., Taylor, R., Florence A. J., Shankland, K. (2010). Characterisation of amorphous and nanocrystalline molecular materials by total scattering. CrystEngComm 12, 1366-1368.

Billinge, S. J. L., Kanatzidis, M. G. (2004). Beyond crystallography: the study of disorder nanocrystallinity and crystallographically challenged materials. Chem. Commun. 2004, 749-760.

Billinge, S. J. L., Levin, I. (2007). The problem with determining atomic structure at the nanoscale. Science 316, 561-565.

Boulahya, K., Gonzalez, L.R., Parras, M., Calbet, J.M.G., Nickolskiy, M.S. \& Nicolopoulos, S. (2007). Ab initio determination of heavy oxide perovskite related structures from precession electron diffraction data. Ultramicroscopy 107, 445-452.

Brown, C. J. (1968). Crystal structure of $\beta$-copper phthalocyanine, J. Chem. Soc. A1968, 2488. 
Capitani, G.C., Oleynikov, P., Hovmöller, S. \& Mellini M. (2006). A practical method to detect and correct for lens distortion in the TEM. Ultramicroscopy 106, 66-74.

Chupas, P. J., Qiu, X., Hanson, J. C., Lee, P. L., Grey, C. P., Billinge, S. J. L. (2003). Rapidacquisition pair distribution function (RA-PDF) analysis. J. Appl. Cryst. 36, 1342-1347.

Cockayne, D.J.H. (2007). The study of nanovolumes of amorphous materials using electron scattering. Annu. Rev. Mater. Res. 37, 159-87.

Coltman, J. W. (1955), Electron diffraction camera, United States Patent 2727153.

Dorset, D.L. (1995). Structural Electron Crystallography, Plenum Publishing Corporation.

Dubochet, J., Adrian, M., Chang, J. J., Homo, J. C., Lepault, J., McDowall, A. W., Schultz, P. (1988). Cryo-electron microscopy of vitrified specimens. Q. Rev. Biophys., 21(2), 129-228.

Dykhne, T., Taylor, R., Florence A., Billinge, S. J. L. (2011). Data requirements for the reliable use of atomic pair distribution functions in amorphous pharmaceutical fingerprinting. Pharm. Res. 28, 1041-1048.

Egami, T., Billinge, S. J. L. (2003). Underneath the Bragg peaks: structural analysis of complex materials. Oxford: Pergamon Press Elsevier.

Elschner, C., Levin, A.A., Wilde, L., Grenzer, J., Schroer, C., Leo, K., Riede, M. (2011). Determining the C60 molecular arrangement in thin films by means of X-ray diffraction. $J$. Appl. Cryst. 44, 983-990.

Farrow, C. L., Billinge, S. J. L. (2009). Relationship between the atomic pair distribution function and small angle scattering: implications for modeling of nanoparticles. Acta Cryst. A65, 232-239.

Farrow, C. L., Juhás, P., Liu, J., Bryndin, D., Bozin, E. S. , Bloch, J., Proffen, T., Billinge, S. J. L. (2007). PDFfit2 and PDFgui: Computer programs for studying nanostructure in crystals. J. Phys: Condens. Mat.19, 335219.

Farrow, C. L., Ruan C.-Y., Billinge, S. J. L. (2010), Quantitative nanoparticle structures from electron crystallography data. Phys. Rev. B81, 124104.

Faruqi, A. R, McMullan, G. (2011). Electronic detectors for electron microscopy. Q. Rev. Biophys. 44, 357-390.

Gemmi, M., Zou, X., Hovmoller, S., Migliori, A., Vennstrom, M. \& Andersson, Y. (2003). Structure of Ti2P solved by three-dimensional electron diffraction data collected with the precession technique and high-resolution electron microscopy. Acta Cryst. A59, 117-126.

Gemmi, M. \& Nicolopoulos, S. (2007). Structure solution with three-dimensional sets of precessed electron diffraction intensities. Ultramicroscopy 107, 483-494. 
Gemmi, M., Voltolini, M., Ferretti, A.M. \& Ponti, A. (2011). Quantitative texture analysis from powder-like electron diffraction data. J. Appl. Cryst. 44, 454-461.

Gorelik, T., Schmidt, M. U., Brüning, J., Bekoe, S. \& Kolb, U. (2009). Using Electron Diffraction to Solve the Crystal Structure of a Laked Azo Pigment. Cryst. Growth Des. 9, 3898-3903.

Gorelik, T., Matveeva, G., Kolb, U., Schleuß, T., Kilbinger, A.F.M., v. de Streek, J., Bohle, A., Brunklaus, G. (2010). H-bonding schemes of di- and tri-p-benzamides assessed by a combination of electron diffraction, X-ray powder diffraction and solid-state NMR. CrystEngComm. 12, 1824-1832.

Gorelik, T.E., van de Streek, J., Kilbinger, A.F.M., Brunklaus, G. \& Kolb, U. (2012). Abinitio crystal structure analysis and refinement approaches of oligo p-benzamides based on electron diffraction data. Acta Cryst. B 68, 171-181.

Herbst, W. \& Hunger, K (2004). Industrial Organic Pigments, 3rd ed., Weinheim: WileyVCh.

Hirata, A., Hirotsu, Y., Ohkubo, T., Hanada, T., Bengus, V. Z. (2006). Compositional dependence of local atomic structures in amorphous Fe 100- $\mathrm{x} \mathrm{B} \mathrm{x}(\mathrm{x}=14,17,20)$ alloys studied by electron diffraction and high-resolution electron microscopy. Phys. Rev. B 74, 214206-1-9.

Hirata, A., Morino, T., Hirotsu, Y., Itoh, K., Fukunaga. T. (2007). Local Atomic Structure Analysis of $\mathrm{Zr}-\mathrm{Ni}$ and $\mathrm{Zr}-\mathrm{Cu}$ Metallic Glasses Using Electron Diffraction. Materials transactions, 48, 1299-1303.

Hirotsu, Y., Ohkubo, T., Bae, I.-T., Ishimaru, M. (2003). Electron diffraction structure analysis for amorphous materials. Materials Chemistry and Physics, 81, 360-363

Juhás, P., Cherba, D. M., Duxbury, P. M., Punch W. F., Billinge, S. J. L. (2006). Ab initio determination of solid-state nanostructure. Nature 440, 655-658.

Juhás, P., Granlund, L., Gujarathi, S. R., Duxbury, P. M., Billinge, S. J. L. (2010). Crystal structure solution from experimentally determined atomic pair distribution functions. $J$. Appl. Cryst. 42, 623-629.

Klug, H. P., Alexander, L. E. (1974). X-ray Diffraction Procedures 2nd Edition, Wiley, New York.

Kolb, U., Gorelik, T., Kübel, C., Otten, M. T., Hubert, D. (2007). Towards automated diffraction tomography: Part I-Data acquisition. Ultramicroscopy 107, 507-513. 
Kolb, U., Gorelik, T., Otten, M. T. (2008). Towards automated diffraction tomography. Part II-Cell parameter determination. Ultramicroscopy 108, 763-772.

Kolb, U., Gorelik, T. E., Mugnailoi, E. \& Stewart, A. (2010). Structural Characterization of Organics Using Manual and Automated Electron Diffraction. Polymer Reviews 50, 385409.

Kolb, U., Mugnaioli, E., Gorelik \& T. E. (2011). Automated electron diffraction tomography - a new tool for nano crystal structure analysis. Cryst. Res. Technol. 46, 542 - 554.

Kim, J.-S., Kim, M.-S., Park, H. J., Jin, S.-J., Lee, S., Hwang, S.-J. (2008). Physicochemical properties and oral bioavailability of amorphous atorvastatin hemi-calcium using spraydrying and SAS process. Int. J. Pharm. 359, 211-219.

Kim, J.G., Seo, J.W., Cheon, J. \& Kim Y.J. (2009). Rietveld Analysis of Nano-crystalline MnFe2O4 with Electron Powder Diffraction. Bull. Korean Chem. Soc. 30, 183-187.

Làbàr, J. L. (2004). Phase identification by combining local composition from EDX with information from diffraction database. In: Electron Crystallography: Novel Approaches for Structure Determination of Nanosized Materials (Eds. T. E. Weirich, J. L. La`ba`r, X. D. Zuo), pp. 207-218, Springer 2006. (Proc. of the NATO Advances Study Institute on Electron Crystallography, Erice, Italy, 10-14 June 2004).

Lábár, J. (2008). Electron diffraction based analysis of phase fractions and texture in nanocrystalline thin films, Part I: Principles. Microsc. Microanal. 14, 287-295.

Lábár, J. L. (2009). Electron Diffraction Based Analysis of Phase Fractions and Texture in Nanocrystalline Thin Films, Part II: Implementation. Microsc. Microanal. 15, 20-29.

Lotsch, B.V., Döblinger, M., Sehnert, J., Seyfarth, L., Senker, J., Oeckler, O. \& Schnick, W. (2007). Unmasking melon by a complementary approach employing electron diffraction, solid-state NMR spectroscopy, and theoretical calculations-structural characterization of a carbon nitride polymer. Chem. Eur. J. 13, 4969 - 4980.

Luo, Z., Vasquez, Y., Bondi, J.F. \& Schaak R.E. (2011). Pawley and Rietveld refinements using electron diffraction from $\mathrm{L}_{2}$-type intermetallic $\mathrm{Au}_{3} \mathrm{Fe}_{1-\mathrm{x}}$ nanocrystals during their in-situ order-disorder transition. Ultramicroscopy 111, 1295-1304.

Marijnissen, J. C. M, Yurteri, C. U., van Erven, J. \& Ciach, T. (2010), Medicine Nanoparticle Production by EHDA, in: Nanoparticles in medicine and environment Inhalation and health effects, Springer. 
McCulloch, D. G., McKenzie, D. R., Goringe, C. M., Cockayne, D. J. H., McBride, W., Green, D. C. (1999). Experimental and Theoretical Characterisation of Structure in Thin Disordered Films. Acta Cryst. A55, 178-187.

Mizuguchi, J., Sasaki, T. \& Tojo, K. (2002). Refinement of the crystal structure of 5,7,12,14tetrahydro[2,3-b]-quinolinoacridine (gamma-form), $\mathrm{C}_{20} \mathrm{H}_{12} \mathrm{~N}_{2} \mathrm{O}_{2}$, at 223 K. Z. Kristallogr. New Crystal Structures 217, 249-250.

Moeck, P., \& Rouvimov, S. (2009). Structural Fingerprinting of Nanocrystals in the Transmission Electron Microscope: Utilizing Information on Projected Reciprocal Lattice Geometry, 2D Symmetry, and Structure Factors, in Drug Delivery Nanoparticles Formulation and Characterization, Informa Healthcare, USA, New York.

Moore, M.D., Steinbach, A.M., Buckner I.S. \& Wildfong, P. L. D (2009). A structural investigation into the compaction behavior of pharmaceutical composites using powder $\mathrm{X}$ ray diffraction and total scattering analysis. Pharm. Res. 26, 2429-2437.

Moss, S. C. \& Graczyk, J. F. (1969). Evidence of voids within the as-deposited structure of glassy silicon. Phys. Rev.Lett. 23, 1167-1171.

Mugnaioli, E., Capitani, G., Nieto, F. and Mellini, M. (2009). Accurate and precise lattice parameters by selected-area electron diffraction in the transmission electron microscope. American Mineralogist, 94, 793-800.

Neder, R. B., Proffen, T. (2009). Diffuse Scattering and Defect Structure Simulations: A Cook Book Using the Program DISCUS. Oxford University Press.

Noerenberg, H., Saeverin, R., Hoppe, U., Holzhueter, G. (1999). Estimation of radial distribution functions in electron diffraction experiments: physical, mathematical and numerical aspects. J. Appl. Cryst. 32, 911-916.

Paulus, E. F., Leusen, F. J. J. \& Schmidt, M. U. (2007). Crystal structures of quinacridones. CrystEngComm 9, 131-143.

Petkov, V., Billinge, S. J. L. (2002), From crystals to nanocrystals: Semiconductors and beyond, in: S. J. L. Billinge and M. F. Thorpe (Eds.), From semiconductors to proteins. Beyond the average structure. [Proceedings of the workshop "From Semiconductors to Proteins: Beyond the Average Structure", helt July28 - August 1, 2001 in Traverse City, Michigan]. Plenum, New York, 2002.

Petkov, V., Parvanov, V., Trikalitis, P., Malliakas, C., Vogt, T. \& Kanatzidis, M. (2005a). Three-dimensional structure of nanocomposites from atomic pair distribution function 
analysis: study of polyaniline and polyaniline ${ }_{0.5} \mathrm{~V}_{2} \mathrm{O}_{5} \times 1.0 \mathrm{H}_{2} \mathrm{O}$. J. Am. Chem. Soc. 127, 8805-8812.

Petkov, V., Parvanov, V., Tomalia, D., Swanson, D., Bergstrom, D. \& Vogt, T. (2005b). 3D structure of dendritic and hyper-branched macromolecules by x-ray diffraction. Solid State Communications, 134, 671-675.

Prasad, R. S., Yandrapu, S. K., Manavalan, R. (2010). Preparation and characterization of itraconazole solid dispersions for improved oral bioavailability. Int. J. ChemTech Res., 2, $133-142$

Rademacher, N., Daemen, L. L., Chronister, E. L., Proffen, T. (2012). Pair distribution function analysis of molecular compounds: significance and modeling approach discussed using the example of p-terphenyl. J. Appl. Cryst. 45, 482-488.

Schmidt, M.U., Brühne, S., Wolf, A.K., Rech, A., Brüning, J., Alig, E., Fink, L., Buchsbaum, C., Glinnemann, J., van de Streek, J., Gozzo, F., Brunelli, M., Stowasser, F., Gorelik, T., Mugnaioli, E., Kolb, U. (2009). Electron Diffraction, X-ray Powder Diffraction and Pair Distribution Function Analyses to Determine the Crystal Structures of Pigment Yellow 213, $\mathrm{C}_{23} \mathrm{H}_{21} \mathrm{~N}_{5} \mathrm{O}_{9}$. Acta Cryst. B, 65, 189-199.

Schmidt, M.U. (2010). PDF investigations on quinacridone polymorphs. 12th European Powder Diffraction Conference EPDIC-12, Darmstadt, Germany, 27-30 August 2010.

Sproul, A., McKenzie, D.R., Cockayne, D.J.H. (1986). Structural study of hydrogenated amorphous silicon-carbon alloys. Phil. Mag. B 54, 113-131.

Tucker, M. G., Keen, D. A., Dove, M. T., Goodwin, A. L.; Hui, Q. (2007). RMCProfile: reverse Monte Carlo for polycrystalline material. J. Phys.: Condensed Matter, 19, 335218/1-335218/16.

Vainshtein, B. K. (1964). Structure Analysis by Electron Diffraction; Pergamon Press: New York.

Vincent, R. \& Midgley., P.A. (1994). Double conical beam-rocking system for measurement of integrated electron diffraction intensities. Ultramicroscopy 53, 271-282.

Voigt-Martin, I.G., Yan, D.H., Yakimansky, A., Schollmeyer, D., Gilmore, C.J. \& Bricogne, G. (1995). Structure determination by electron crystallography using both maximumentropy and simulation approaches. Acta Cryst. A51, 849-868.

Wagner, C. N. J. (1978). Direct methods for the determination of atomic-scale structure of amorphous solids (X-ray, electron, and neutron scattering). J. Non-Cryst. Solids 31, 1.

Warren, B. E. (1969). X-ray Diffraction. Addison-Wesley, New York. 
Waseda, Y. (1980). The Structure of Non-crystalline Materials. McGraw-Hill, New York

Weirich, T .E., Winterer, M., Seifried, S., Hahn, H. \& Fuess, H. (2000). Rietveld analysis of electron powder diffraction data from nanocrystalline anatase, $\mathrm{TiO}_{2}$. Ultramicroscopy 81, 263-270.

Weirich, T. E., Portillo, J., Cox, G., Hibst, H. \& Nicolopoulos, S. (2006). Ab initio determination of the framework structure of the heavy-metal oxide $\mathrm{Cs}_{\mathrm{X}} \mathrm{Nb}_{2.54} \mathrm{~W}_{2.46} \mathrm{O}_{14}$ from $100 \mathrm{kV}$ precession electron diffraction data. Ultramicroscopy 106, 164-175.

Wolf, A. K., Brühne, S., Glinnemann, J., Hu, C., Kirchner, M. T., Schmidt, M. U. (2012). Local atomic order in sodium p-chlorobenzenesulfonate monohydrate studied by pair distribution function analyses and lattice-energy minimisations. Z. Krist. 127, 113-121.

Wright, A. C. (1985). Scientific opportunities for the study of amorphous solids using pulsed neutron sources. J. Non-Cryst. Solids 76, 187.

Young, C. A., Goodwin, A. L. (2011). Applications of pair distribution function methods to contemporary problems in materials chemistry. J. Mater. Chem. 21, 6464-6476.

Yu, L. (2001). Amorphous pharmaceutical solids: Preparation, characterization and stabilization. Advanced Drug Delivery Reviews, 48, 27-42.

Zewail, A. H. (2006). 4D ultrafast electron diffraction, crystallography, and microscopy. Annu. Rev. Phys. Chem. 57, 65-103.

Zhang, P., Borgnia, M., Mooney, P., Shi, D., Pan, M., O'Herron, P., Mao, A., Brogan, D., Milne, J.L.S. \& Subramaniam, S (2003). Automated image acquisition and processing using a new generation of $4 \mathrm{~K}$ x $4 \mathrm{~K}$ CCD cameras for cryo electron microscopic studies of macromolecular assemblies. J. Struct. Biol. 143(2), 135-144. 